\title{
Evaluation of the Impact of Static Interference on an Empirical Data Based Static Magnetic Localization Setup for Capsule Endoscopy
}

https://doi.org/10.1515/cdbme-2020-3017

\begin{abstract}
In this paper, the impact of interference due to the geomagnetic field on a static magnetic localization setup for capsule endoscopy, which is suitable for a wearable application, was investigated. For this purpose, a study was carried out in which the average abdomen size of 15 subjects was evaluated. With the determined geometry values, a setup consisting of three elliptical sensor rings was modeled. Simulations were performed, where the magnetic flux density was evaluated at the sensors by using different-sized magnets. The measured values were compared with each other and the geomagnetic flux density. The results revealed that the measured values were for all evaluated magnet sizes of the order of the geomagnetic flux density, which is problematic since the calibration of sensors is no longer valid if the orientation of the wearable sensor array is changed. However, it is suggested that a differential measurement is suitable for the proposed system and could reduce static interference caused by the geomagnetic field.
\end{abstract}

Keywords: capsule endoscopy, geomagnetic field, magnetic localization, wireless capsule endoscopy.

\section{Introduction}

The conventional method for the diagnosis and treatment of diseases of the gastrointestinal tract (GIT) is endoscopy. During the examination, a flexible tube is guided through the GIT to the location of interest. Since the GIT is about $9 \mathrm{~m}$ long [1],

${ }^{*}$ Corresponding author: Samuel Zeising, Institute for Electronics Engineering, Friedrich-Alexander-Universität Erlangen-Nürnberg (FAU), Cauerstr. 9, Erlangen, Germany, E-Mail: samuel.zeising@fau.de

Daisuke Anzai, Nagoya Institute of Technology, Graduate School of Engineering, Nagoya, Japan

Angelika Thalmayer, Institute for Electronics Engineering, Friedrich-Alexander-Universität Erlangen-Nürnberg (FAU), Erlangen, Germany

Georg Fischer, Institute for Electronics Engineering

Friedrich-Alexander-Universität Erlangen-Nürnberg (FAU),

Erlangen, Germany

Jens Kirchner, Institute for Electronics Engineering, FriedrichAlexander-Universität Erlangen-Nürnberg (FAU), Erlangen, Germany it would be desirable to know the location of interest in advance. Furthermore, the conventional procedure requires anesthesia and can be perceived as unpleasant by the patient. Wireless capsule endoscopy (WCE) provides a less invasive alternative and thus could tackle these problems; capsules for endoscopy are up to $32 \mathrm{~mm}$ long and have a diameter of about $12 \mathrm{~mm}$. Therefore, they are suitable to be swallowed. Their main components are a camera, a battery, a printed circuit board, and an antenna for transmitting a video stream. For further diagnosis and treatment, each video frame must be correlated with the exact traveled distance of the capsule in the GIT to determine the location of interest. [2]

A promising method to tackle this problem is the static magnetic localization [2-6]. Therefore, a permanent magnet is embedded in the capsule; the resulting static magnetic flux density is measured vectorially by magnetic sensors arranged in arrays outside of the body.

Considering that it takes the capsule about 8 hours to travel through the GIT, the patient cannot be expected to remain in a fixed position during the examination. Therefore, to enable the patient to continue daily routine during the application of WCE, the sensor system must be wearable, compact and robust towards static interference of the geomagnetic field. In [4], a bulky cuboid wearable system with 16 sensors was designed with 2 additional sensors for canceling interference of the geomagnetic field, whereas in [5], a wearable system consisting of 3 rings with 96 sensors was proposed. In the latter, however, it was not stated which size the sensor array had. The proposed size of the permanent magnet for localization varies widely in the literature, e.g. in [4], a magnet with size of $15 \times 10 \mathrm{~mm}$ (diameter $\times$ length) was used, whereas in [5], a magnet of size $6 \times 12 \mathrm{~mm}$, respectively. A smaller magnet was implemented in [7] of a size $5 \times 6 \mathrm{~mm}$. In [3, 5], it was not stated which magnet size was used. However, the used magnet size as well as dimensions of the sensor array matter for the localization performance, since the magnitude of the magnetic flux density of a permanent magnet is determined by the magnet size and decreases rapidly with distance and the space for a magnet in a capsule is limited due to the built-in components. If the magnitude of the flux density of the magnet becomes comparable to that of the geomagnetic field, the latter significantly disturbs the localization process. With the patient 
moving during WCE, this leads to a time-varying interference, not only a static offset.

In this paper, the impact of interference of the geomagnetic field on a wearable system for the localization of endoscopy capsules was investigated in COMSOL. An approach for prevention of interference caused by the static geomagnetic field, which is suitable for the proposed setup, was suggested.

\section{Methods}

\subsection{Static Magnetic Localization}

A well-established method for the localization of capsules for endoscopy is the static magnetic localization [2-6]. A magnet of length $l$, radius $k$ and magnetization $M_{0}$ (along the longitudinal axis of the magnet) in ampere per meter, is embedded in a capsule which is swallowed by a subject. By arranging magnetic sensors on the abdomen of a subject, a magnetic flux density $B\left(x_{i}, y_{i}, z_{i}\right)$ is measured at the $i$ th sensor. The proposed magnetic sensor array system was established with three identical, stable and elliptical rings with the diameters $d_{1}$ and $d_{2}$ (Fig. 1) and four magnetic sensors were mounted on each ring. The rings had a distance of $h / 2$ from each other. The number and distribution of the sensors are optimization parameters for the position and orientation error [6]. However, this work was focused on the evaluation of the impact of the geomagnetic field on the proposed system, therefore, optimization was not within the scope of this study.

The magnetic flux density of a permanent magnet for the $i$ th sensor can be approximated as a magnetic dipole if the distance from the magnet to the $i$ th sensor is much larger than the geometry of the magnet. The magnetic dipole model is then described by [7]

$$
B\left(x_{i}, y_{i}, z_{i}\right)=\frac{\mu_{0} \mu_{\mathrm{r}} M_{0} l \pi k^{2}}{4 \pi}\left(\frac{3\left\langle H_{0}, P_{i}\right\rangle P_{i}}{R_{i}{ }^{5}}-\frac{H_{0}}{R_{i}^{3}}\right),
$$

where $P_{i}$ indicates the vector from the center of the permanent magnet $P_{\text {mag }}=(a, b, c)^{\top}$ to the position of the $i$ th sensor $S_{i}=\left(x_{i}, y_{i}, z_{i}\right)^{\top}$, where $\hat{B}\left(x_{i}, y_{i}, z_{i}\right)$ is measured. Moreover, $H_{0}=(m, n, p)^{\top}$ is the normalized orientation vector of the permanent magnet and $R_{\mathrm{i}}=\left\|S_{i}-P_{\mathrm{mag}}\right\|_{2}$ is the Euclidean distance between the center of the magnet to the $i$ th sensor. The permeability in vacuum is expressed by $\mu_{0}=4 \pi \times 10^{-7} \mathrm{Vs} / \mathrm{Am}$ and the relative permeability $\mu_{\mathrm{r}}$ of the human body and free space are approx. equal.

For the estimation of the position $(a, b, c)^{\top}$ and orientation $(m, n, p)^{\top}$ of the magnet, the respective components of the measured magnetic flux density $\hat{B}\left(x_{i}, y_{i}, z_{i}\right)$ at the $i$ th sensor are subtracted from the corresponding analytical components of $B\left(x_{i}, y_{i}, z_{i}\right)$. In this way, an error function is determined

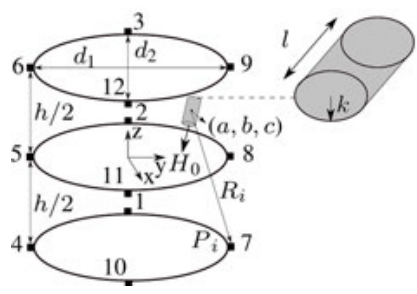

Fig. 1: Scenario of the static magnetic localization method. The sensors are arranged in rings.

and by minimizing it according to the parameters $a, b, c, m, n$ and $p$, the position and orientation of the magnet are estimated.

One specific drawback of this method is that it is prone to the interference from static magnet fields. For Central Europe, the $x$ - (north), $y$ - (west), and $z$ - (vertical)-components of the geomagnetic flux density $B_{\text {geo }}$ are about $(20,-1,-45)^{\top} \mu \mathrm{T}$, which leads to an absolute value of $49 \mu \mathrm{T}$ with a worldwide variation of $25 \mu \mathrm{T}$ to $65 \mu \mathrm{T}$ [8].

\subsection{Geometry of the Localization Setup}

For the static magnetic localization, sensors have to be arranged around the body. Sensors arranged in rings are eligible for that purpose. Therefore, the dimensions of the human abdomen were estimated. Thereby, the geometry of the sensor setup, which can be described with the parameters $d_{1}, d_{2}$ and $h$ (Fig. 1), can be determined. Therefore, a study population of 15 subjects ( 3 females and 12 males) was considered. Care was taken to ensure that the body-mass index (BMI) of the test subjects varied widely. For each subject, the BMI, the distance $d_{2}$ from the stomach to the back, the large diameter $d_{1}$ and the height of the abdomen were measured and the average values concerning the subjects were determined. Based on these values, the localization setup was modeled in COMSOL.

\subsection{Evaluation of the Measured Magnetic Flux Density}

The material of the permanent magnet was set to neodymiumiron-boron $(\mathrm{NdFeB})$ with grade $\mathrm{N} 52$ since that material yields the highest remanence-to-volume ratio for permanent magnets [9]. The corresponding magnetization $M_{0}$ was approx. $1140 \mathrm{kA} / \mathrm{m}$ and was set along the longitudinal axis of the magnet. Considering the maximal length and diameter of a capsule are around $32 \mathrm{~mm}$ and $12 \mathrm{~mm}$, respectively. Therefore, the magnet size has to be adapted. With the information on the average dimensions of the human abdomen, 12 sensors were arranged in three elliptical rings and as a next step, the whole localization setup was simulated. Different magnet sizes were 
considered and at first, the absolute value of $\hat{B}$ was evaluated concerning the distance from the magnet and compared with

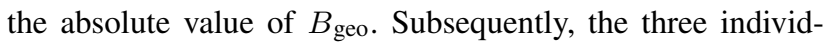
ual components of $\hat{B}$ at the respective sensor positions were evaluated. Therefore, the different magnets were placed in the representative position of $(50,50,50)^{\top} \mathrm{mm}$ (see Fig. 1 for the coordinate system), which covers realistic distances from all sensors. The orientation of the magnet was set to $(1,0,0)^{\top}$, which was also the orientation of $M_{0}$. The three components of $\hat{B}$ were evaluated at the known sensor positions and compared with the three components of $B_{\text {geo }}$.

\section{Results}

\subsection{Study of the Abdomen Size}

The average BMI of the subjects was $26.4 \pm 4.3$. The result for the average distance from the stomach to the back $d_{2}$ was $26 \pm 4 \mathrm{~cm}$. The average value of the parameter $d_{1}$ was $35 \pm 3 \mathrm{~cm}$. For the height of the abdomen $h$, the average value was $17 \pm 2 \mathrm{~cm}$. Therefore, three identical elliptical sensor rings with $d_{1}=40 \mathrm{~cm}$ and $d_{2}=33 \mathrm{~cm}$ were proposed (Fig. 1). A tolerance of $7 \mathrm{~cm}$ was added to the average value of $d_{2}$. Consequently, the movement of the abdomen during breathing and the standard deviation of $d_{2}$ were considered. For $d_{1}$, a tolerance of $5 \mathrm{~cm}$ to the average value was added. The average abdomen height plus its standard deviation was $19 \mathrm{~cm}$. Thus, to cover most of the abdomen and, in consequence, the GIT, the distance $h / 2$ from one ring to another, was set to $10 \mathrm{~cm}$.

\subsection{Evaluation of the Measured Flux Density}

The absolute value of $\hat{B}$, produced by a cylindrical permanent magnet, concerning the distance to the magnet, was evaluated. The $|\hat{B}|$ decays approx. as $1 / R^{3}$ with distance. The results for different lengths and radii are depicted in Fig. 2. A distance of about $9 \mathrm{~cm}$ to $20 \mathrm{~cm}$ to the magnet led to a $|\hat{B}|$, which had the magnitude of $\left|B_{\text {geo }}\right|$ in all the evaluated cases. This holds true, even if the variation of the geomagnetic field worldwide $(25 \mu \mathrm{T}$ to $65 \mu \mathrm{T})$ is considered. The higher the volume of the magnet, the higher is the $|\hat{B}|$, in terms of the distance.

Taken into account the geometry of the sensor rings and the position of the magnet $(50,50,50)^{\top} \mathrm{mm}$ and that 12 sensors were arranged in three elliptical rings as depicted in Fig. 1 , there was a distance from the center of the magnet to the respective sensors in the interval of $13.5-29.6 \mathrm{~cm}$. In Table 1, the average absolute values of the three components of $\hat{B}$ at

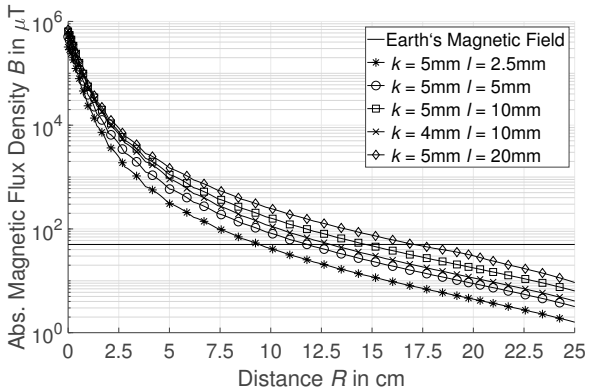

Fig. 2: $|\hat{B}|$ concerning the distance from the magnet. $\left|B_{\text {geo }}\right|$ is compared with the $|\hat{B}|$ of different magnet sizes. The $x$ - and $y$-axis are in linear- and logarithmic-scale, respectively.

the sensors, for different magnet sizes, are shown. For all eval-

Tab. 1: Average values of the three components of the measured $\hat{B}$ for different magnet sizes.

\begin{tabular}{lccc}
\hline Magnet Size $k \times l$ & $\varnothing \hat{B}_{\mathrm{x}}$ in $\mu \mathrm{T}$ & $\varnothing \hat{B}_{\mathrm{y}}$ in $\mu \mathrm{T}$ & $\varnothing \hat{B}_{\mathrm{z}}$ in $\mu \mathrm{T}$ \\
\hline $5 \times 2.5 \mathrm{~mm}$ & $3.4 \pm 2.7$ & $2.4 \pm 2.5$ & $2.1 \pm 2.5$ \\
$5 \times 5 \mathrm{~mm}$ & $6.8 \pm 5.4$ & $4.8 \pm 5.0$ & $4.2 \pm 4.9$ \\
$5 \times 10 \mathrm{~mm}$ & $13.5 \pm 10.9$ & $9.6 \pm 10.0$ & $8.5 \pm 9.9$ \\
$4 \times 10 \mathrm{~mm}$ & $8.7 \pm 7.2$ & $6.1 \pm 6.4$ & $5.5 \pm 6.3$ \\
$5 \times 20 \mathrm{~mm}$ & $27.1 \pm 21.7$ & $19.2 \pm 20.1$ & $17.1 \pm 19.9$ \\
\hline
\end{tabular}

uated cases, the average $x$-component was higher than the $y$ and $z$-component, this was expected since the orientation of $H_{0}$ was set to $(1,0,0)^{\top}$. The standard deviation was for all three components of the order of the average value, which indicates a huge difference for the values at the individual sensors. This is due to the position of the magnet. Fig. 3 shows the three components of $\hat{B}$, for a magnet with a length and diameter of $10 \mathrm{~mm}$, concerning the respective sensors and the components of $B_{\text {geo }}$. As expected, the sensors 8-12 with a distance of

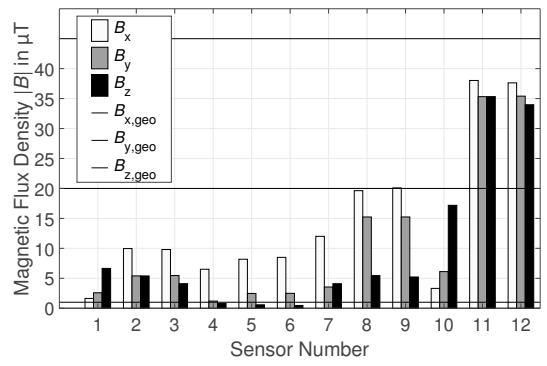

Fig. 3: $\hat{B}\left(x_{i}, y_{i}, z_{i}\right)$ for a magnet with length and diameter of $10 \mathrm{~mm}$. The horizontal lines are the values of $B_{\text {geo }}$.

$13.5-19.6 \mathrm{~cm}$ from the magnet had the highest measured values. Sensor 4 was approx. $30 \mathrm{~cm}$ away from the magnet and 
its components were still in the $\mu \mathrm{T}$-range. No measured value was higher than the $z$-component of $B$ geo. The $y$-component of $B$ geo was exceeded for all sensors, whereas the $x$-component only for sensors 11 and 12 . Overall, the individual components of the measured $\hat{B}$ were for each sensor and magnet size in the $\mu$ T-range and, therefore, had the magnitude of $B$ geo.

\section{Discussion and Outlook}

By evaluating the average abdomen size of the study population, a circular sensor array system was designed. Due to its elliptical shape and its lower amount of sensors compared with $[4,5]$, the system is more suitable for a wearable application. The three components of the magnetic flux density $\hat{B}$ at the sensors were evaluated and compared, for different magnet sizes, with the components of $B_{\text {geo }}$. Assuming that one-third of the space inside the capsule is used for the magnet, a magnet length of $2.5-10 \mathrm{~mm}$ appears reasonable. The radius of a capsule is approx. $6 \mathrm{~mm}$, considering its enveloping plastic layer, a radius $k$ of $4-5 \mathrm{~mm}$ is appropriate.

The magnetic flux density, of a magnet of that size, would be detectable at the proposed sensor positions, for state-of-theart magnetic sensors. According to [10], the $B$ of the magnet must be at least four times higher than $B_{\text {geo }}$ at the sensors to get an average position error below $10 \mathrm{~mm}$. Since the measured $\hat{B}(x, y, z)$ and $B_{\text {geo }}$, for all evaluated magnet sizes, were both in the $\mu \mathrm{T}$-range, elimination of the inference of $B_{\text {geo }}$ is mandatory, especially if the orientation of the sensors is changed during the examination.

In 2019, Shao et al. [4] proposed a method of preventing interference caused by the geomagnetic field. For this purpose, two additional magnetic sensors, mounted on the back and chest, were used. It was assumed that a distance of approx. $20 \mathrm{~cm}$ to the sensor array, would lead to a neglectable value for the measured magnetic flux density at the additional sensors compared with the value measured at the sensor array. Therefore, it was assumed that by subtracting the values of the additional sensors from the values of the sensor array, the static interference would be eliminated. However, the results of this paper show that even if the distance from the magnet reached about $30 \mathrm{~cm}$, there was still a measured flux density, which was in the $\mu \mathrm{T}$-range and, therefore, had an impact on the localization error. Thus, it is concluded that Shao's approach is dependent on the position of the magnet because as the magnet travels in the lower region of the GIT and, thus, further away from the additional sensors, the error by applying that method should be decreasing. Furthermore, the additional sensors were not part of the sensor array; thus, the relative position regarding the coordinate system of the sensor array was not stable and the orientation of the additional sensors can vary highly, if the patient's spine is not straight, e. g. during sitting, from those of the sensors used for localization.

In the future, a differential measurement will be conducted. For this purpose, the sensors will be divided into sensor pairs, each consisting of two identically oriented sensors. Since the geomagnetic field is equal for such sensor pairs, subtracting the measured magnetic flux density of the two sensors, from which the positions are known, from each other, the static disturbance will be reduced. Thus, no additional sensors, which are not part of the array, will be needed and the system will be more stable. Another interesting method of prevention of magnetic interference is the signal space separation (SSS) method which will be investigated in the future [11]. Moreover, number and distribution of the sensors will be optimized.

\section{Author Statement}

Research funding: The authors state no funding was involved. Conflict of interest: The authors state no conflict of interest.

\section{References}

[1] Kent G. Human Anatomy. 5th ed. McGraw-Hill College 2000.

[2] Mateen M, Basar R, Ahmed A and Ahmad M. Localization of Wireless Capsule Endoscope: A Systematic Review. IEEE Sensors Journal, vol. 17, no. 5, pp. 1197-1206, 2017.

[3] Wang M, Shi Q, Song S, Hu C, Meng, M. A Novel Relative Position Estimation Method for Capsule Robot Moving in Gastrointestinal Tract. Sensors, vol. 19, 2019.

[4] Shao G, Tang Y, Tang L, Dai Q and Guo Y. A Novel Passive Magnetic Localization Wearable System for Wireless Capsule Endoscopy. IEEE Sensors Journal, vol. 19, no. 9, pp. 3462-3472, 2019.

[5] Hu C et al. Locating Intra-Body Capsule Object by ThreeMagnet Sensing System. IEEE Sensors Journal, vol. 16, no. 13, pp. 5167-5176, 2016.

[6] Kanaan M and Cil M. Cramer-Rao Lower Bounds for Magnetic Localization of a Wireless Capsule Endoscope based on the Magnetic Dipole Model. International Symposium on Personal, Indoor and Mobile Radio Communications, 2019.

[7] Jackson J. Classical Electrodynamics. 1st ed. New York: John Wiley \& Sons 1962.

[8] National Centers for Environmental Information, 2020. Accessed on: March 04, 2020. [Online]. Available: https://www.ngdc.noaa.gov/

[9] Mukhopadhyay S C and Gupta G S. Smart Sensors and Sensing Technology. 1st ed. Berlin Heidelberg: SpringerVerlag 2008.

[10] Shao G and Guo Y, "An Optimal Design for Passive Magnetic Localization System Based on SNR Evaluation," in IEEE Transactions on Instrumentation and Measurement, vol. 69, no. 7, pp. 4324-4333, 2020.

[11] Taulu S, Simola J and Kajola M, "Applications of the signal space separation method," in IEEE Transactions on Signal Processing, vol. 53, no. 9, pp. 3359-3372, 2005. 